\title{
A PESQUISA EXPERIMENTAL SOBRE CO- MUNICAÇÃO DE UTILIDADE PÚBLICA: UMA REVISÃO SISTEMÁTICA
}

\author{
WLADIMIR GRAMACHO \\ UNIVERSIDADE DE BRASÍLIA \\ BRASÍLIA, DISTRITO FEDERAL, BRASIL \\ WGGRAMACHO@GMAIL.COM \\ EMILLY RAMOS BEHNKE \\ UNIVERSIDADE DE BRASÍLIA \\ BRASÍLIA, DISTRITO FEDERAL, BRASIL \\ EMILLYBEHNKE@GMAIL.COM \\ VITÓRIA CAVALCANTI DE SOUZA \\ UNIVERSIDADE DE BRASÍLIA \\ BRASÍLIA, DISTRITO FEDERAL, BRASIL \\ VITORIA.SOUZACAVALCANTI@GMAIL.COM
}




\section{A PESQUISA EXPERIMENTAL SOBRE COMUNICAÇÃO DE UTILIDADE PÚBLICA: UMA REVISÃO SISTEMÁTICA}

Resumo: $O$ artigo analisa as principais características de estudos experimentais sobre comunicação de utilidade pública divulgados em 2016 por periódicos classificados no estrato A1 do Qualis/Capes nas áreas de comunicação, ciência política, psicologia e saúde coletiva. Dentre os 38 estudos selecionados, foi mais comum a análise dos efeitos de textos sobre intenções de comportamento.

Palavras-chave: Metodologia experimental; Comunicação pública; Comunicação em Saúde; Revisão sistemática.

\section{LA INVESTIGACIÓN EXPERIMENTAL SOBRE COMUNICACIÓN DE UTILIDAD PÚBLICA: UNA REVISIÓN SISTEMÁTICA}

Resumen: Se analizan las características de estudios experimentales sobre comunicación de utilidad pública publicados en 2016 por revistas A1 (Qualis) Capes) en las áreas de comunicación, ciencia política, psicología y salud colectiva. Entre los 38 estudios seleccionados, fueron más comunes la medición de efectos de mensajes sobre las intenciones de comportamiento.

Palabras Clave: Metodología experimental; Comunicación pública; Comunicación en salud; Revisión sistemática.

\section{THE EXPERIMENTAL RESEARCH ON PUBLIC SERVICE COMMUNICA-}

\section{TION: A SYSTEMATIC REVIEW}

Abstract: The paper analyzes the main characteristics of experimental studies on public service communication published in 2016 by journals classified in the A1 stratum of Qualis/Capes in the fields of communication, political science, psychology and public health. Among the 38 selected studies, it was more common to find analysis of text manipulations in participants' behavior intentions.

Keywords: Experimental methodology; Public communication; Health Communication; Systematic review.

\section{INTRODUÇÃO}

O prêmio Nobel de economia de 2019 consagrou não só um grupo de pesquisadores mas, particularmente, uma metodologia de investigação científica. Abhijit Banerjee, Esther Duflo (ambos do MIT) e Michael Kremer (Harvard) foram recompensados por seu estudo sobre políticas públicas mais efetivas na melhoria das condições de vida de pessoas em situação de 
extrema pobreza no mundo. Mas os elogios acabaram sendo especialmente dirigidos à revolução metodológica conduzida por eles no âmbito da pesquisa sobre desenvolvimento econômico ao introduzirem "estudos experimentais cuidadosamente desenhados", que demonstraram ser "abordagens poderosas, como o uso de experimentos de campo", para usar expressões da nota oficial à imprensa divulgada pela The Royal Swedish Academy of Sciences.

Apesar da crescente relevância da metodologia experimental em áreas como a economia, a psicologia ou a ciência política, sua aplicação é pouco difundida no campo da comunicação, em que ensaios e raros estudos empíricos de caráter observacional ainda respondem pela maioria da produção acadêmica, especialmente no Brasil. Não é surpreendente, portanto, que essa abordagem metodológica seja ainda mais rara numa área mais específica da comunicação, como nos estudos sobre a comunicação de utilidade pública, onde são mais comuns manuais com recomendações extraídas do senso comum ou de práticas consolidadas. Um levantamento na plataforma Scielo com os termos de busca "metodologia experimental" e "comunicação de utilidade pública” (e suas possíveis variações terminológicas), por exemplo, não encontrará um artigo sequer nessa área de conhecimento. Nos anos recentes, alguns estudos surgiram numa área de conhecimento próxima, como nos estudos de opinião sobre políticas públicas, como nos exemplos de Turgeon et al. (2014), sobre cotas raciais numa universidade pública, e de Mundim et al. (2019), sobre o programa Bolsa Família.

Apesar dessa lacuna, a inauguração de uma agenda consistente de estudos experimentais no âmbito da comunicação de utilidade pública poderia ter repercussões científicas e aplicadas muito positivas no país. Sob a perspectiva acadêmica, a metodologia experimental supera as limitações de determinação causal dos estudos observacionais. Isso porque o método experimental, por meio da manipulação de variáveis independentes e do controle do contexto pesquisado, permite observar efeitos causais sobre as variáveis dependentes de interesse. Neste sentido, os experimentos apresentam amplo potencial de contribuição científico-empírica na comunicação ao oferecer um método idôneo e robusto para o teste de hipóteses e a discussão de teorias que, com frequência, restringem-se às perspectivas normativa ou qualitativa.

Já no âmbito da agenda pública, o uso de métodos experimentais pode esclarecer, por exemplo, o desenho mais apropriado de mensagens em 
campanhas de conscientização sobre comportamentos que impõem riscos à saúde, opiniões que podem ameaçar a sustentabilidade ambiental ou atitudes que despertam animosidade entre diferentes grupos sociais. Sob essa perspectiva aplicada, o conhecimento produzido por estudos experimentais na comunicação pública podem ser úteis para dar mais eficiência a decisões relevantes como a escolha de enquadramentos narrativos, de expressões específicas em mensagens, de porta-vozes, da identidade visual de peças gráficas e audiovisuais, ou da identidade sonora de peças audiofônicas, para citar alguns exemplos.

Este artigo, portanto, ocupa-se de descrever as características metodológicas dos estudos sobre efeitos de comunicação de utilidade pública baseados em evidências experimentais publicados em periódicos de excelência acadêmica internacional. A resposta a esta questão não pretende oferecer um manual para a realização de estudos experimentais, mas descrever as principais práticas adotadas pelas pesquisas de ponta na área. Interessam aqui, portanto, características como as relacionadas à escolha e medição de variáveis dependentes, à manipulação de variáveis independentes de maior interesse, à seleção dos participantes, aos contextos e recursos da pesquisa e às técnicas mais frequentes de análise de dados, entre outras. O escopo temporal da amostra analisada foi o ano de 2016 e os periódicos considerados foram os do estrato A1 no Qualis/Capes, que privilegiam a maior qualidade em cada área de conhecimento.

Este artigo está organizado em três seções adicionais. A primeira delas explica o método adotado nesta revisão de literatura, composto por quatro etapas: seleção de artigos, definição do protocolo, aplicação do protocolo e análise dos dados. A segunda seção apresenta e discute os resultados deste trabalho, distinguindo achados relacionados a três unidades de análise: os artigos selecionados, os estudos experimentais publicados em cada artigo (alguns artigos divulgaram mais de um estudo) e as hipóteses testadas. Finalmente, a última seção apresenta as principais conclusões deste artigo e indica possíveis tópicos de uma agenda futura de pesquisa na área.

\section{METODOLOGIA}

A metodologia de trabalho desta pesquisa pode ser resumida em quatro etapas. Na primeira, foram definidos os parâmetros para a seleção da amostra de artigos a serem analisados para responder à pergunta de pesquisa indicada anteriormente. Os parâmetros definidores da amostra 
foram (i) a qualidade dos periódicos a serem contemplados na busca, (ii) as áreas de conhecimento que com alguma frequência se ocupam do estudo sobre comunicação de utilidade pública, (iii) o escopo temporal da busca e (iv) a opção pelo uso da metodologia experimental em cada artigo. A seleção amostral definitiva contemplou trabalhos publicados em periódicos classificados como A1 pelo Qualis/Capes, estrato mais elevado desse ranqueamento. A intenção deste recorte foi analisar somente a produção científica com melhores avaliações e, assim, mapear um repertório de excelência acadêmica. As áreas de conhecimento incluídas foram: Comunicação e Informação; Ciência Política e Relações Internacionais; Psicologia; e Saúde Coletiva. São esses os campos de estudo que, em diferentes proporções, ocupam-se da investigação de efeitos da comunicação midiática, em sentido amplo, e também da comunicação aplicada a problemas de utilidade pública. $\mathrm{Na}$ área de comunicação e informação, foram retirados do escopo de avaliação as publicações relacionadas à biblioteconomia, editoração e ciência da informação. Em relação à ciência política e às relações internacionais, não foram analisados os periódicos sobre demografia e relações internacionais, em geral. Já sobre as publicações de psicologia e saúde coletiva, foram eliminados os periódicos de caráter mais técnico ou direcionado a problemas específicos de cada área, como, por exemplo, aqueles relacionados à medicina neurológica. Restaram, portanto, os periódicos com temáticas que de algum modo poderiam ter relação com estudos associados à comunicação de utilidade pública. Além disso, não foram incluídos na análise da pesquisa os periódicos com edições semanais (com mais de 50 edições anuais, portanto) ou com número superior a 100 artigos em cada edição, o que extrapolaria a capacidade de trabalho dedicada a este projeto. Ainda assim, apenas seis periódicos foram descartados com base nesses dois critérios de exclusão: BMC Health Services Research, BMC Public Health, Lancet (edição britânica), Nature (Londres), Plos One e Proceedings Of The National Academy Of Sciences Of The United States Of America.

Quanto ao corte temporal, optou-se por analisar apenas os trabalhos publicados nas edições impressas do ano de 2016. Ou seja, ainda que tenham aparecido nas versões digitais das revistas em 2015, tomamos como referência a data de capa da versão impressa. O ano de 2016 era o mais recente quando foi iniciado o processo de leitura e classificação dos artigos, ao final do ano de 2017. Dentre os artigos selecionados com base nesses critérios, procedeu-se a uma leitura dos resumos para avaliar cada um deles quanto à 
adesão ao tema de interesse (i.e., estudos de comunicação midiática de utilidade pública) e à metodologia experimental. Nos periódicos de temas pertinentes, os artigos foram selecionados a partir do uso do termo "experim" como filtro de busca dos resumos de cada estudo. A seleção final resultou em 29 artigos científicos publicados em periódicos A1 no ano de 2016 que tratavam de temas de comunicação de utilidade pública com utilização de metodologia experimental. O número pode parecer pequeno, por um lado, mas por outro reflete o ainda incipiente uso desta metodologia numa área de conhecimento aplicada da comunicação, com grande relevância para a implementação de políticas públicas e programas governamentais. A Tabela 1 resume o processo de seleção amostral e indica o número de artigos e estudos contemplados em cada uma das áreas.

Tabela 1: Áreas de conhecimento e etapas na seleção amostral de artigos e estudos

\begin{tabular}{|c|c|c|c|c|c|}
\hline & $\begin{array}{c}\text { Saúde } \\
\text { coletiva }\end{array}$ & Psicologia & $\begin{array}{c}\text { Comunicação e } \\
\text { Informação }\end{array}$ & $\begin{array}{l}\text { Ciência } \\
\text { pol. e RI }\end{array}$ & Total \\
\hline Periódicos no Qualis & $\begin{array}{c}345 \\
(49 \%)\end{array}$ & $\begin{array}{c}200 \\
(28 \%)\end{array}$ & $\begin{array}{c}55 \\
(8 \%)\end{array}$ & $\begin{array}{l}103 \\
(15 \%)\end{array}$ & $\begin{array}{c}703 \\
(100 \%)\end{array}$ \\
\hline $\begin{array}{l}\text { Periódicos A1 analisa- } \\
\text { dos, devido à temáti- } \\
\text { ca pertinente à comu- } \\
\text { nicação midiática }\end{array}$ & $\begin{array}{c}13 \\
(8 \%)\end{array}$ & $\begin{array}{c}68 \\
(41 \%)\end{array}$ & $\begin{array}{c}32 \\
(19 \%)\end{array}$ & $\begin{array}{c}54 \\
(32 \%)\end{array}$ & $\begin{array}{c}167 \\
(100 \%)\end{array}$ \\
\hline $\begin{array}{l}\text { Artigos selecionados, } \\
\text { devido ao uso de } \\
\text { metodologia exper- } \\
\text { imental em estudos } \\
\text { sobre comunicação } \\
\text { de utilidade pública }\end{array}$ & $\begin{array}{c}21 \\
(72 \%)\end{array}$ & $\begin{array}{c}6 \\
(21 \%)\end{array}$ & $\begin{array}{c}2 \\
(7 \%)\end{array}$ & $\begin{array}{c}0 \\
(0 \%)\end{array}$ & $\begin{array}{c}29 \\
(100 \%)\end{array}$ \\
\hline Estudos analisados & $\begin{array}{c}27 \\
(71 \%)\end{array}$ & $\begin{array}{c}8 \\
(21 \%)\end{array}$ & $\begin{array}{c}3 \\
(8 \%)\end{array}$ & $\begin{array}{c}0 \\
(0 \%)\end{array}$ & $\begin{array}{c}38 \\
(100 \%)\end{array}$ \\
\hline
\end{tabular}

Fonte: Elaboração dos autores.

A segunda etapa desta pesquisa consistiu na definição do protocolo de classificação, com base em referências de boas práticas no uso de metodologia experimental, como as discutidas por McDermott (2002) e Mutz (2011). Nesta fase, foram definidas três unidades de análise, que descreveriam características (i) dos artigos, (ii) dos estudos publicados nos artigos e (iii) das hipóteses testadas nos estudos. A elaboração do protocolo resultou em sete variáveis a serem observadas sobre os artigos, 36 variáveis 
relacionadas aos estudos e uma única variável sobre as hipóteses (se elas tiveram apoio nos dados ou não). A Tabela 2 descreve as variáveis em cada unidade de análise.

Tabela 2: Variáveis incluídas no protocolo de análise

\begin{tabular}{|c|l|}
\hline $\begin{array}{c}\text { Unidade de } \\
\text { análise }\end{array}$ & \multicolumn{1}{c|}{ Variáveis } \\
\hline Artigo & $\begin{array}{l}\text { Número do artigo, área de conhecimento, número de estudos publica- } \\
\text { dos no artigo, número de autores (incluindo homens e mulheres), pre- } \\
\text { sença de autoras, número de autoras (mulheres) e número de páginas } \\
\text { ocupadas pelo artigo. }\end{array}$ \\
\hline Estudos & $\begin{array}{l}\text { Número do estudo, tipo de variável dependente, tipo de variável ma- } \\
\text { nipulada, referência a alguma teoria, teoria mencionada, número de } \\
\text { hipóteses testadas no estudo, ambiente do experimento, uso de real- } \\
\text { ismo experimental quando o experimento foi realizado no laboratório, } \\
\text { técnica de designação aleatória dos participantes às condições exper- } \\
\text { imentais, tipo de participantes, técnica de seleção dos participantes, } \\
\text { número total de participantes, número de mulheres participantes, } \\
\text { idade média dos participantes, número de condições experimentais, } \\
\text { tamanho médio dos grupos, duração do experimento, publicação de } \\
\text { dados descritivos sobre os participantes, publicação dos estímulos, in- } \\
\text { formação sobre a inserção do estímulo num contexto midiático mais } \\
\text { amplo, descrição sucinta do contexto midiático, desenho do experi- } \\
\text { mento, uso de placebo, referência a checagem de manipulação, relato } \\
\text { de viés do pesquisador, relato de viés de expectativa, relato de viés de } \\
\text { demanda, tipo de mensuração, tipo de incentivos oferecidos aos partic- } \\
\text { ipantes, valor dos incentivos (quando monetários), relato de ameaças à } \\
\text { validade interna, relato de ameaça à validade externa devido ao uso de } \\
\text { uma amostra não probabilística, uso de declaração de consentimento, } \\
\text { uso de engano, esclarecimento do engano ao final da pesquisa quando } \\
\text { utilizado e técnica de análise de dados. }\end{array}$ \\
\hline Hipóteses & \begin{tabular}{l} 
Amparada ou refutada pelos dados. \\
\hline
\end{tabular} \\
\hline
\end{tabular}

Fonte: Elaboração dos autores.

Na terceira etapa, o protocolo foi aplicado pelas duas autoras deste artigo e as classificações foram revisadas pelo autor. Em caso de dúvida ou discordância entre os três, foram discutidos em detalhe os trechos dos artigos que deveriam esclarecer o dado a ser registrado na planilha. As divergências, entretanto, foram raras, uma vez que as variáveis do protocolo demandavam informações bastante objetivas. A quarta e última etapa da pesquisa consistiu na revisão e análise do banco de dados, extraindo estatísticas descritivas univariadas e bivariadas das variáveis indicadas no pro- 
tocolo.

\section{RESULTADOS}

Esta seção descreve os principais resultados obtidos neste levantamento com relação às características das três unidades de análise contempladas (artigos, estudos e hipóteses), que dão ordem às subseções seguintes. Ao final da seção, a Tabela 3 resume as médias e frequências das principais variáveis utilizadas na codificação dos estudos. Além dos achados apresentados aqui, outras informações podem ser obtidas no banco de dados, disponível por meio de solicitação ao autor correspondente.

\subsection{Características dos artigos}

Em primeiro lugar, foi notável a concentração de artigos numa das quatro áreas de conhecimento contempladas nesta pesquisa. Dentre os 29 artigos analisados, 21 (72\%) pertenciam à área de saúde coletiva. Esse resultado antecipa uma característica dos estudos aqui analisados, que acabaram se concentrando em tópicos da área de saúde, dentre os vários que compõem esforços aplicados de comunicação pública, como segurança, educação ou meio ambiente. Em seguida, residualmente, foram encontrados textos em revistas das áreas de psicologia (seis, ou 21\%) e comunicação (dois, ou 7\%). Em 2016, periódicos da área de ciência política não publicaram artigos sobre a temática em análise.

Em média, os artigos contaram com 3,8 autores por texto, indicando trabalho coletivo sobre o objeto desta revisão, em muitos casos resultado de redes de cooperação entre diferentes instituições de ensino. Quanto ao gênero de autores, quase um quarto dos artigos (sete, ou 24\%) foram escritos só por homens e exatamente a mesma proporção foi escrita só por mulheres. Do total de autores (109), 49\% eram mulheres e 51\%, homens, o que sugere um equilíbrio de gêneros de pesquisadores experimentalistas interessados em comunicação de utilidade pública, especificamente no ano pesquisado e nos periódicos avaliados.

Em média, os artigos tinham 11 páginas (mínimo de seis e máximo de 25), dedicadas a uma narrativa científica sucinta, organizada, em geral, em torno a uma discussão bastante específica do marco teórico (quando existente), à descrição das características do experimento e à análise dos resultados e suas consequências práticas. Quanto à referência a teorias, mais especifica- 
mente, foi surpreendente observar que a maioria dos estudos (16, ou 55\%) não estava ancorada explicitamente em nenhum modelo explicativo.

A grande maioria dos artigos (21, ou 72\%) publicou um único experimento. Entretanto, em sete artigos foram publicados dois estudos e um deles publicou três (Zhao et al, 2016, sobre o combate ao fumo). No total, portanto, foram publicados 38 estudos experimentais, que consistirão na unidade de análise da subseção seguinte.

\subsection{Características dos estudos}

Um dos aspectos mais importantes nas campanhas de comunicação pública é a definição do seu objetivo, ou como preferem Rice \& Atkin (2001), do comportamento focal a ser influenciado pelas mensagens e seus porta-vozes. Consequentemente, a decisão sobre a variável dependente dos estudos é crucial para a avaliação da potencial influência que a comunicação pode ter sobre os comportamentos a serem estimulados em campanhas de promoção ou a serem evitados em campanhas de prevenção.

A análise da amostra desta pesquisa identificou dez níveis da variável dependente escolhida pelos pesquisadores, sendo que o estudo das intenções de comportamento foi a mais prevalente, contemplada em $63 \%$ dos estudos (e.g. King, 2016, sobre câncer de pele; Lee \& Park, 2016, sobre o vírus H1N1, e Popova et al., 2016, sobre o combate ao fumo). Em menor medida, os experimentos também analisaram a influência de diferentes tratamentos sobre as opiniões, o que ocorreu em 39\% dos casos (e.g., Sun et al., 2016, sobre obesidade; e Hoek et al, 2016, sobre fumo), e sobre as atitudes, presentes em 34\% dos estudos (e.g., Chen et al, 2016, sobre persuasão; e Mutti et al, 2016, sobre fumo). Outras variáveis dependentes analisados foram o comportamento efetivo dos participantes (Schultz et al, 2016, sobre controle no uso de água), sua atenção (Occa \& Suggs, 2016, sobre câncer de mama), seu nível de conhecimento após os estímulos (Occa \& Suggs, 2016, idem), suas emoções (e.g., Andrews et al, 2016, sobre cigarros; e Gendall et al., 2016, também sobre fumo), seu engajamento na elaboração após a exposição a um conteúdo (King, 2016, sobre câncer de pele), sua memória (idem) e suas crenças (Zhao, 2016, sobre tabaco). Normalmente, cada estudo tinha mais de uma variável resposta, o que dá conta de um esforço por analisar a influência de campanhas de comunicação em diferentes âmbitos.

Como estímulos de comunicação, ou variáveis independentes de inte- 
resse, o repertório dos experimentos analisados contemplou seis elementos. Provavelmente por motivos de simplicidade operacional na condução dos experimentos, o mais comum foi a manipulação de textos (em $71 \%$ dos casos, como em Chang, 2016, sobre o consumo e cálcio; Knobloch-Westerwick., 2016, sobre o desejo de engravidar; e McCormick \& Seta, 2016, sobre o processamento de mensagens com diferentes enquadramentos). Outros estímulos também investigados exploraram diferenças na seleção de imagens (32\% dos estudos, como em Lazard et al., 2016, sobre saúde mental; ou em Mabry \& Turner, 2016, sobre o enfrentamento às agressões sexuais) e a estratégia narrativa em vídeo (21\%, como em Zhao, 2016, sobre fumo; ou em Gillig \& Murphy, 2016, sobre o apoio a jovens LGBTQ). Finalmente, apenas um estudo ocupou-se da decisão sobre o meio de divulgação (Schultz et al, 2016, analisaram a diferença entre campanhas pela web ou com contato interpessoal em esforços para reduzir o consumo de água), e outro dedicou-se a analisar duas decisões importantes na construção da mensagem: uma sobre a porta-voz e outra sobre a utilização de vídeo ou infografia (Occa \& Suggs, 2016, que observaram seus efeitos em campanha para prevenir o câncer de mama entre mulheres).

Os contextos em que ocorreram os experimentos foram sobretudo dois: os clássicos estudos de laboratório (18, ou 47\%) e os cada vez mais comuns estudos aplicados via internet (17, ou $45 \%$ ). Apenas cinco pesquisas (13\%) foram conduzidas como estudos de campo (Schultz et al., 2016; Mutti et al., 2016; Brewer et al., 2016; e King, 2016), em que os tratamentos ocorrem fora do laboratório, em ambiente natural de interação humana e social, nos quais pode-se reivindicar maior validade externa (Mutz, 2011; Banerjee \& Duflo, 2017). Dentre os estudos de laboratório, nenhum dos trabalhos analisados procurou dar algum realismo aos procedimentos realizados, como o uso de simuladores para a performance de atividades. Entretanto, dois deles incluíram seus estímulos em contextos midiáticos mais amplos. Um dos estudos ambientou um anúncio numa revista online (Knobloch-Westerwick, 2016) e o outro manipulou a posição do mostruário de cigarro numa loja de conveniência virtual (Nonnemaker, 2016).

Quanto ao desenho, a grande maioria dos estudos (32, ou 84\%) optou pelo formato between-subjects, ou entre sujeitos, em que cada participante é submetido a apenas uma condição experimental. O segundo desenho mais comum foram as tarefas de classificação, ou rating tasks, com apenas quatro estudos (11\%), realizados por Hoek et al. (2016), Cathcart e Glendon 
(2016) e Gendall et al. (2016), que executaram dois estudos desse tipo, em que os participantes são convidados a avaliar diferentes conjuntos de mensagens - por exemplo - dentre um número muito maior de mensagens, com o objetivo de medir, por exemplo, qual delas tem maior eficiência na obtenção de determinado resultado de comunicação. Um único estudo, de Lee \& Park (2016), optou pelo formato intra-sujeito, ou within-subject, em que cada participante é submetido a mais de uma condição experimental.

Entre os estudos between-subjects, o número de condições experimentais foi, em média, de 3,9, com mínimo de 2 grupos e máximo de 12 grupos. Já o número de participantes nos estudos teve média de 440 indivíduos, com mínimo de 68 e máximo de 2.083 pessoas. Essa média, entretanto, foi muito diferente entre os experimentos conduzidos em laboratório $(X=211,3)$ e os online $(X=590,9)$. O número médio de participantes em cada condição experimental também variou bastante, de 71,4 nos estudos laboratoriais a 206,6 dentre aqueles realizados online. A facilidade de acesso a um número muito maior de pessoas é um dos principais atrativos dos experimentos feitos pela internet, o que se reflete nos números de participantes recrutados nessa modalidade, em comparação com estudos laboratoriais. No total a média de participantes por grupo - ou condição experimental - foi de 113 indivíduos.

Os estudos, em geral, não traziam informações sobre a duração dos procedimentos experimentais, desde o estímulo à mensuração dos resultados. Dentre os oito que trouxeram essa informação, entretanto, o mais rápido durou 7 minutos e meio (Gendall et al., 2016, sobre fumo) e o mais demorado durou 10 dias (Kknobloch-Westerwick, 2016, sobre o desejo de engravidar), com exposição prolongada a um conteúdo online.

Três tipos de participantes foram mais comuns: adultos em geral participaram de 17 estudos (45\%), como em Sapiains et al. (2016), sobre efeitos de enquadramento no debate sobre mudanças climáticas; estudantes universitários, que com frequência formam amostras de conveniência em pesquisas acadêmicas, foram objeto de 14 estudos (37\%) como em Pfattheicher et al. (2016), sobre proteção ambiental; e outros sete trabalhos (18\%) se dedicaram a analisar adolescentes e crianças, como em Vasiljevic et al. (2016), sobre cigarros eletrônicos com sabor de bala.

Os estudos com esses três grupos de participantes tiveram características bastante diferentes em alguns quesitos. Pesquisas com crianças e adolescentes contaram com indivíduos com idade média de 17,1 anos, em 
estudos com número bastante elevado de participantes $(X=1049,0)$, devido sobretudo à opção por coletas online. Já trabalhos feitos com adultos recrutaram indivíduos com média de idade de 32,6 anos, em número menor de participantes, com média de 421,2 por estudo. Finalmente, pesquisas com estudantes universitários tinham indivíduos com média de idade de 21,5 anos, mas com média bastante inferior de participantes no total $(X=155,1)$, em boa medida por adotarem pesquisas presenciais em laboratório, que têm como vantagem maior controle sobre a administração dos tratamentos, a atenção dos participantes e a mensuração dos resultados.

Entre os estudos que informaram os gêneros dos participantes (33, ou $87 \%$ ), foi possível identificar que pouco mais da metade (52\%) eram mulheres. Um deles, entretanto, não contou com nenhuma mulher devido à natureza da pesquisa de Mabry \& Turner (2016), que estudaram efeitos de mensagens sobre a probabilidade de que homens manifestem a intenção de intervir para evitar atos de violência sexual contra mulheres. Outros três estudos contaram apenas com a participação de mulheres, também devido ao objeto das respectivas pesquisas (Knobloch-Westerwick et al., 2016, sobre desejo de engravidar; Occa e Suggs, sobre prevenção ao câncer de mama; e McCormick \& Seta, 2016, sobre vacinação de crianças contra sarampo, caxumba e rubéola, ainda que neste caso coubesse também estudar as reações dos homens).

Em relação à seleção dos participantes de cada experimento, é importante destacar inicialmente que nenhum deles utilizou uma amostra representativa, o que dá conta de uma dificuldade entre os pesquisadores em dar maior validade externa a trabalhos que, em geral, privilegiam sua validade interna. Amostras de conveniência foram o mais comum, presentes em 20 estudos (53\%), em que os pesquisadores fizeram uso, por exemplo, de plataformas como a M-Turk, da empresa Amazon.com, Inc.. A auto-seleção a partir de um convite aberto deu origem a nove estudos (24\%), outros seis (16\%) foram feitos com combinação de amostras de conveniência e auto-seleção, e três estudos recrutaram participantes interceptando-os em pontos de fluxo.

Alguns critérios importantes para avaliar a qualidade das pesquisas experimentais são a observação e discussão da existência de vieses que podem ameaçar a validade interna dos estudos. Entretanto, em nenhum dos trabalhos analisados seus autores relataram ou discutiram a existência de vieses como os do pesquisador, de expectativas e/ou de demanda. Em geral, 
os autores tampouco discutiram a existência de ameaças à validade interna dos estudos realizados. Cinco pesquisas (13\%) relataram alguma desconfiança de que o trabalho poderia ter perdido sua validade interna, como em Kim \& Shapiro (2016), por exemplo, que reportaram um possível problema nas instruções aos participantes sobre a leitura dos textos manipulados, considerando que deveriam ter sido mais enfáticas. Já o relato de limitações de validade externa foi bastante mais comum, presente em 23 estudos (61\%).

Informações adicionais ou complementares aos dados e modelos estatísticos foram pouco frequentes também. Dados descritivos dos participantes dos experimentos foram publicados pelos pesquisadores em 14 estudos (37\%); os estímulos administrados foram publicados em apenas 15 trabalhos (39\%) - ainda que boa parte deles tenha manipulado textos e não vídeos ou arquivos sonoros -; e nenhum dos estudos publicou o questionário utilizado.

$\mathrm{O}$ uso de placebo foi muito raro entre os trabalhos analisados. Apenas os estudos de Mabry \& Turner (2016)., sobre estratégias para enfrentar agressões sexuais, e de Popova et al. (2016), sobre esforços para reduzir o fumo entre cadetes da força aérea americana, aplicaram placebo a uma das condições experimentais, de modo a controlar os efeitos mensurados nos grupos tratados.

Por outro lado, o uso de incentivos esteve muito mais presente nos estudos aqui selecionados, sendo observado em 23 casos (61\%). As recompensas mais comuns foram pagamentos em dinheiro, utilizadas em 17 trabalhos (45\%), como nos de Rosenberg \& Siegel (2016), sobre efeitos de anúncios da indústria farmacêutica que promovem o consumo de drogas. Em dez estudos (26\%), os autores informaram os valores dos incentivos, que variaram de US\$ 0,40 a US\$185 (Brewer et al., 2016, sobre alertas em maços de cigarro). Outros incentivos também utilizados foram créditos para estudantes de graduação, cupom para sorteio de prêmio e brindes in-kind como camisetas ou chocolate.

A checagem de manipulação curiosamente foi adotada poucas vezes. Esse é um dos passos mais importantes na realização de experimentos, pois assegura que os participantes notaram o elemento manipulado, ainda que não saibam que esse elemento é o objeto de manipulação da pesquisa. Apesar de sua relevância para a indicação de causalidade, a realização dessa checagem foi informada em apenas 10 estudos (26\%). Se os demais trabaIhos a fizeram, não relataram o procedimento.

Dentre as três formas de mensuração de variáveis resposta descritas por 
McDermott (2002), a mais predominante foi o uso de questionários autopreenchidos, presencialmente ou online, observados em 36 estudos (95\%). As exceções foram Mutti et al. (2016), em que os questionários tiveram a intermediação de um(a) aplicador(a), e Schultz et al. (2016), que observaram o consumo de água dos participantes, portanto seu comportamento efetivo.

Outro procedimento importante da pesquisa experimental é o uso de declaração de consentimento, também conhecido como Termo de Consentimento Livre e Esclarecido (TCLE), em que o participante diz ter sido informado sobre a existência de qualquer risco ou desconforto durante a pesquisa e declara estar de acordo com sua exposição ao tratamento. A maioria dos estudos 20 dos 38 (53\%) mencionou o uso dessa declaração, que nem sempre foi citada em trabalhos cujos participantes eram adultos ou estudantes universitários, mas que foi explicitamente informada em todas as pesquisas em que os sujeitos eram crianças ou adolescentes, portanto indivíduos menores de idade.

O uso de engano - quando o participante é induzido a pensar algo diferente do que é o real objeto do estudo ou é oferecida informação não verdadeira - foi relatado em apenas sete casos (18\%). Quando o engano é utilizado, recomenda-se seu esclarecimento ao final do experimento (debriefing), o que foi relatado em apenas dois estudos (5\%), publicados no artigo de Koot et al. (2016), que analisou a relação do consenso entre as fontes de uma notícia e atitudes sobre o meio ambiente. $O$ uso do engano, entretanto, apresenta sérias questões éticas, discutidas, por exemplo, no livro organizado por Teele (2014) sobre usos e abusos em pesquisas experimentais.

Diversas técnicas estatísticas foram usadas na análise dos dados obtidos, mas as mais frequentes foram ANOVA (Análise de Variância) e ANCOVA (Análise de Covariância), presentes em 19 estudos (50\%), seguidas por MANOVA (Análise Multivariada de Variância) e MANCOVA (Análise Multivariada de Covariância), presentes em seis estudos (ou 16\%), Teste t (16\%) e regressões lineares, logística, logit e outras (13\%), para citar as técnicas mais comuns.

Outros elementos importantes sobre a transparência dos estudos realizados são as publicações de dados sociodemográficos dos participantes, realizadas em 14 estudos (37\%), a apresentação dos estímulos introduzidos no experimento, presentes em 15 estudos (39\%) e a reprodução de ao menos parte do questionário, de modo a permitir uma avaliação da redação e ordem das perguntas, o que não foi feito em nenhum dos trabalhos analisa- 
dos.

\subsection{Características das hipóteses}

Um último dado de interesse na análise dos artigos desta revisão de literatura trata das hipóteses de trabalho testadas pelos experimentos. Em primeiro lugar, vale registrar que seis dos 38 estudos (16\%) sequer mencionaram hipóteses, o que revela um interesse muito mais aplicado do que acadêmico em alguns dos trabalhos. Entre os estudos que, sim, mencionaram hipóteses, a média de expectativas indicadas foi de 3,4 por artigo. No total, 110 hipóteses formuladas foram testadas, para as quais foram encontradas evidências empíricas favoráveis em $66 \%$ dos casos. Com estes dados, não é possível dizer se houve um viés na publicação majoritária de estudos cujas hipóteses foram apoiadas pelos resultados, mas vale observar um registro mais ou menos balanceado de hipóteses nulas rejeitadas ou não, o que mostra disposição dos periódicos para publicar também artigos cujas hipóteses de trabalho não tiveram apoio empírico.

A Tabela 3 resume os principais resultados da pesquisa e descreve o perfil médio/modal dos estudos experimentais sobre comunicação em temas de utilidade pública. A análise dos artigos publicados em revistas do estrato A1 do Qualis/Capes em 2016 sobre comunicação de utilidade pública, que utilizaram metodologia experimental, revelou alguns padrões bastante marcados nos 38 estudos analisados. Em primeiro lugar, a área de saúde coletiva foi, notadamente, a que mais se ocupou em produzir conhecimento sobre esse tema. Em apenas 13 periódicos elegíveis, foram encontrados 21 artigos, muito mais que nas demais áreas de conhecimento analisadas.

Os trabalhos majoritariamente estudaram os efeitos de diferentes características de textos sobre as intenções de comportamento dos participantes, medidas por meio de questionários autopreenchidos. A partir das médias e frequências observadas, pode-se descrever o desenho médio/ modal das pesquisas nessa área como estudos between-subjects, em que os participantes são escolhidos em amostras de conveniência, mediante recompensa com pagamento em dinheiro, e aleatoriamente designados para quatro condições experimentais, com aproximadamente 50 indivíduos por grupo quando os estudos são feitos em laboratório, ou 150 indivíduos quando ocorrem via internet.

Entre as características de qualidade ou éticas analisadas, foram notá- 
veis a ausência de discussões sobre possíveis ameaças à validade interna dos estudos, a falta de checagens de manipulação e escassos relatos sobre a existência de algum viés que possa ter influenciado os resultados. Apesar disso, praticamente metade dos estudos informou haver utilizado uma declaração de consentimento, firmada pelos participantes ou seus responsáveis legais, no caso de menores de idade.

Tabela 3: Principais resultados da análise dos 29 artigos (38 estudos) selecionados

\begin{tabular}{|c|c|}
\hline $\begin{array}{l}\text { Artigos publicados, por } \\
\text { área de conhecimento }\end{array}$ & Saúde coletiva (72\%), Psicologia (21\%) e Comunicação (7\%). \\
\hline Autores & Média de 3,8 por artigo; no total, $49 \%$ mulheres, $51 \%$ homens. \\
\hline Variável dependente $^{1}$ & $\begin{array}{l}\text { Intenção de comportamento (63\% dos estudos), opinião } \\
\text { (39\%) e atitude (34\%). }\end{array}$ \\
\hline Forma de mensuração & $\begin{array}{c}\text { Questionários autopreenchidos (95\%), questionários aplica- } \\
\text { dos (3\%) e observação de comportamento (3\%). }\end{array}$ \\
\hline Variável manipulada ${ }^{1}$ & $\begin{array}{c}\text { Textos (71\%), imagem (32\%) e estratégia narrativa em vídeo } \\
(21 \%) .\end{array}$ \\
\hline Contexto ambiental & $\begin{array}{c}\text { Estudos de laboratório ( } 47 \%) \text {, estudos via internet (45\%) e } \\
\text { estudos de campo (13\%). }\end{array}$ \\
\hline Desenho experimental & $\begin{array}{l}\text { Between-subjects (84\%), rating tasks (11\%) e within-subject } \\
(3 \%) .\end{array}$ \\
\hline $\begin{array}{c}\text { Condições } \\
\text { experimentais }{ }^{2}\end{array}$ & Média de 3,9 condições experimentais por estudo. \\
\hline $\mathrm{N}$ dos estudos & $\begin{array}{l}\text { Média de } 440 \text { indivíduos por estudo, sendo que a média foi } \\
\text { de } 211 \text { nos estudos feitos em laboratório e de } 590 \text { nos estu- } \\
\text { dos online. }\end{array}$ \\
\hline $\mathrm{N}$ dos grupos & $\begin{array}{l}\text { Média de } 113 \text { por estudo, sendo que a média foi de } 71 \text { in- } \\
\text { divíduos em estudos laboratoriais e de } 206 \text { nos estudos } \\
\text { online. }\end{array}$ \\
\hline Tipos de participantes & $\begin{array}{l}\text { Adultos (45\%), estudantes universitários (37\%) e crianças ou } \\
\text { adolescentes (18\%). }\end{array}$ \\
\hline Idade dos participantes & $\begin{array}{c}\text { Média de 32,6 anos nos estudos com adultos, de } 21,5 \text { anos } \\
\text { com estudantes universitários e de } 17,1 \text { anos com crianças e } \\
\text { adolescentes. }\end{array}$ \\
\hline $\begin{array}{l}\text { Gênero dos } \\
\text { participantes }\end{array}$ & Mulheres (52\%) e homens (48\%). \\
\hline Amostras & $\begin{array}{l}\text { Amostra de conveniência ( } 53 \% \text { ), auto-seleção (24\%) e difer- } \\
\text { entes combinações (16\%). }\end{array}$ \\
\hline Incentivos & Pagamentos em dinheiro (45\%), créditos (5\%) e brindes (5\%). \\
\hline $\begin{array}{l}\text { Checagem de } \\
\text { manipulação }\end{array}$ & $26 \%$ dos estudos informaram a realização dessa checagem. \\
\hline
\end{tabular}




\begin{tabular}{|c|c|}
\hline $\begin{array}{c}\text { Declaração de } \\
\text { consentimento }\end{array}$ & $53 \%$ dos estudos mencionaram a utilização dessa declaração. \\
\hline Uso de engano & $\begin{array}{c}18 \% \text { dos estudos relataram o uso do engano, sendo que ape- } \\
\text { nas } 5 \% \text { informaram a realização de debreafing. }\end{array}$ \\
\hline Análise de dados & $\begin{array}{c}\text { ANOVA e ANCOVA (50\%), MANOVA e MANCOVA (16\%) e teste } \\
t(16 \%) .\end{array}$ \\
\hline
\end{tabular}

Fonte: Elaboração dos autores.

Notas:

${ }^{1}$ Os estudos normalmente tinham mais de uma variável dependente ou manipulada, por isso a soma das frequências supera os $100 \%$.

${ }^{2}$ Entre os desenhos between-subjects.

\section{CONSIDERAÇÕES FINAIS}

A amostra analisada neste artigo oferece informações bastante estruturantes para a inauguração de uma agenda de pesquisa no país. Estudos experimentais em comunicação de utilidade pública poderiam oferecer oportunidades empíricas de teste de teorias dedicadas a prescrever e discutir efeitos de comunicação midiática, bem como incentivariam a produção de conhecimento aplicado de modo a apoiar ações e campanhas de promoção ou prevenção de comportamentos, atitudes ou opiniões que estimulem o bem-estar dos indivíduos e sua convivência pacífica.

Nesse sentido, tópicos importantes para pesquisas futuras no país podem ser a identificação de mensagens e porta-vozes mais eficientes para estimular a vacinação contra graves doenças, para promover o combate ao mosquito Aedes aegypti (transmissor de doenças como dengue, zika e chikungunya), para incentivar denúncias contra a violência de gênero ou racial, para reduzir o consumo de água, ou para esclarecer procedimentos de coleta seletiva do lixo, entre outros. Nos últimos anos, alguns desses problemas da agenda pública brasileira têm sido temas de ações ou campanhas de comunicação que investiram milhões de reais na compra de mídia, mas raras vezes se ocuparam de pré-testar suas ações de modo a otimizar investimentos, reduzir as chances de efeitos bumerangue e observar as mudanças sociais esperadas. A utilização de metodologia experimental nesses casos pode representar uma revolução, como a descrita pela The Royal Swedish Academy of Sciences a respeito do trabalho de Banerjee, Duflo e Kremer. 


\section{REFERÊNCIAS}

ANDREWS, J., NETEMEYER, R., BURTON, S., KEES, J. Effects of plain package branding and graphic health warnings on adolescent smokers in the USA, Spain and France. Tobacco Control, London, v. 25, n. e2, p. e120-e126, 2016.

BANERJEE, A.V.; DUFLO, E. (Eds) Handbook of Economic Field Experiments. Vol 1. Oxford: North-Holland, 2017.

BREWER, N., HALL, M., LEE, J., PEEBLES, K., NOAR, S., RIBISL, K. Testing warning messages on smokers' cigarette packages: a standardised protocol. Tobacco Control, London, v. 25, n. 2, p. 153-159, 2016.

CATHCART, R. L., GLENDON, A. I. Judged effectiveness of threat and coping appraisal anti-speeding messages. Accident Analysis \& Prevention, Amsterdam, v. 96, p. 237-248, 2016.

CHANG, C. Behavioral Recommendations in Health Research News as Cues to Action: Self-Relevancy and Self-Efficacy Processes. Journal of Health Communication: International Perspective, Nova York, v. 21, n. 8, p. 954-968, 2016

CHEN, M., BELL, R. A., TAYLOR, L. D. Narrator Point of View and Persuasion in Health Narratives: The Role of Protagonist-Reader Similarity, Identification, and Self-Referencing. Journal of Health Communication: International Perspective, Nova York, v. 21, n. 8, p. 908-918, 2016.

DRUCKMAN, J; LEEPER, T. Learning more from political communication experiments: pretreatment and its effects. America Political Science Review, Cambridge, v. 56, n. 4, p. 875-896, 2012.

DRUCKMAN, J.; PETERSON, E.; SLOTHUUS, R. How Elite Partisans Polarization Affects Public Opinion Formation., America Political Science Review, Cambridge, v. 107, n. 1, p. 57-79, 2013.

GENDALL, P., ECKERT, C., HOEK, J., FARLEY, T., LOUVIERE, J., WILSON, N., EDWARDS, R. Estimating the 'consumer surplus' for branded versus standardised tobacco packaging. Tobacco Control, London, v. 25, n. 6, p. 641-647, 2016.

GERBER, A.S.; GREEN, D.P. Field Experiments: Design, Analysis, and Interpretation. Nova York: W. W. Norton \& Company, Inc. 2012

GILLIG, T. K., MURPHY, S. T. Fostering Support for LGBTQ Youth? The Effects of a Gay Adolescent Media Portrayal on Young Viewers. International Journal of Communication, Los Angeles, v. 10, p. 3828-3850, 2016.

GRABE, M.E.; WESTLEY, B.H. "The Controlled Experiment," in Mass Communication Research and Theory. Boston: Allyn \& Bacon, 2003, p. 267-298.

GREITEMEYER, T.; OSSWALD, S. Effects of Prosocial Video Games on Prosocial Behavior. Journal of Personality and Social Psychology, Washington, v. 98, n. 2, p. 211-221, 2010.

HOEK, J., GENDALL, P., ECKERT, C., KEMPER, J., LOUVIERE, J. Effects of brand variants on smokers' choice behaviours and risk perceptions. Tobacco Control, London, v. 25, n. 2, p. 160-165, 2016. 
KIM, H. K., SHAPIRO, M. A. When Bad Things Happen to a Protagonist Like You: The Role of Self in Resistance to Negatively Framed Health Narratives. Journal of Health Communication: International Perspective. Nova York, v. 21, n. 12, p. 1227-1235, 2016.

KING, A. J. Visual Exemplification and Skin Cancer: The Utility of Exemplars in Promoting Skin Self-Exams and Atypical Nevi Identification. Journal of Health Communication: International Perspective, Nova York, v. 21, n. 7, p. 826-836, 2016.

KNOBLOCH-WESTERWICK, S., WILLIS, L. E., KENNARD, A. R. Media Impacts on Women's Fertility Desires: A Prolonged Exposure Experiment. Journal of Health Communication, Oxfordshire, v. 21, n. 6, p. 647-657, 2016.

KOOT, C., MORS, E. TER, ELLEMERS, N., DAAMEN, D. D. L. Facilitation of attitude formation through communication: how perceived source expertise enhances the ability to achieve cognitive closure about complex environmental topics. Journal of Applied Psychology, Washington, v. 46, n. 11, p. 627-640, 2016.

LAZARD, A., BAMGBADE, B., SONTAG, J., BROWN, C., Using Visual Metaphors in Health Messages: A Strategy to Increase Effectiveness for Mental Illness Communication. Journal of Health Communication: International Perspective, Nova York, v. 21, n. 12, p. 1260-1268, 2016.

LEE, H., PARK, SA. Third-Person Effect and Pandemic Flu: The Role of Severity, Self-Efficacy Method Mentions, and Message Source. Journal of Health Communication: International Perspective, Nova York, v. 21, n. 12, p. 1244-1250, 2016.

MABRY, A., TURNER, M. M. Do Sexual Assault Bystander Interventions Change Men's Intentions? Applying the Theory of Normative Social Behavior to Predicting Bystander Outcomes. Journal of Health Communication: International Perspective, Nova York, v. 21, n. 3, p. 276-292, 2016.

MCCORMICK, M., SETA, J. J. Lateralized goal framing: How health messages are influenced by valence and contextual/analytic processing. Psychology \& Health, Oxfordshire, v. 31, n. 5, 535-548, 2016.

MCDERMOTT, Rose. Experimental methods in political science. Ithaca: Annual Review of Political Science, Palo Alto, v. 5, p. 31-61, 2002.

MUNDIM, P., VIDIGAL, R., MICHELOTTI, F., PINTO, A. (2019). Bolsa Família, informação e preconceito: uma análise com o uso de experimentos. Revista do Serviço Público, Brasília, v. 70, n. 4, p. 551-575, 2019.

MUTTI, S., REID, J., GUPTA, P., PEDNEKAR, M., DHUMAL, G.,NARGIS, N., HUSSAIN, A., HAMMOND, D. Perceived effectiveness of text and pictorial health warnings for smokeless tobacco packages in Navi Mumbai, India, and Dhaka, Bangladesh: findings from an experimental study. Tobacco Control, London, v. 25, n. 4, p. 437-443, 2016.

NONNEMAKER, J., KIM, A., SHAFER, P., LOOMIS, B., HILL, E., HOLLOWAY, J., FARRELLY, $M$. Influence of point-of-sale tobacco displays and plain black and white cigarette packaging and advertisements on adults: Evidence from a virtual store experimental study.

Addictive Behaviors, Amsterdam, v. 56, p. 15-22, 2016.

OCCA, A., SUGGS, S. Communicating Breast Cancer Screening With Young Women: An 
CADERNOS DE COMUNICAÇÃO

UNIVERSIDADE FEDERAL DE SANTA MARIA

Experimental Test of Didactic and Narrative Messages Using Video and Infographics. Journal of Health Communication: International Perspectives, Nova York, v. 21, n. 1, p. 1-11, 2016.

PFATTHEICHER, S., SASSENRATH, C., SCHINDLER, S. Feelings for the Suffering of Others and the Environment: Compassion Fosters Proenvironmental Tendencies. Environment and Behavior, Thousand Oaks, v. 48, n. 7, p. 929-945, 2016.

POPOVA, L., LINDE, B., BURSAC, Z., TALCOTT, G., MODAYIL, M., LITTLE, M., LING, P., GLANTZ, S., KLESGES, R. Testing antismoking messages for Air Force trainees. Tobacco Control, London, v. 25, n. 6, p. 656-663, 2016.

RICE, Ronald. E.; ATKIN, Charles K. Public Communication Campaigns. Michigan University: Sage Publications, 2001.

ROSENBERG, B. D., JASON, T. S. The Effect of Inconsistency Appeals on the Influence of Direct-to-Consumer Prescription Drug Advertisements: An Application of Goal Disruption Theory. Journal of Health Communication, Oxfordshire, v. 21, n. 2, p. 217-227, 2016.

SAPIAINS, R., BEETON, R. J. S., WALKER, I. A. Individual responses to climate change: Framing effects on pro-environmental behaviors. Journal of Applied Social Psychology, Nova Jersey, v. 46, n. 8, p. 483-493, 2016.

SCHULTZ, P., MESSINA, A., TRONU, G., LIMAS, E., GUPTA, R., ESTRADA, M. Personalized Normative Feedback and the Moderating Role of Personal Norms: A Field Experiment to Reduce Residential Water Consumption. Environment and Behavior, Thousand Oaks, v. 48, n. 5, p. 686-710, 2016.

SUN, Y., KRAKOW, M., JOHN, K. K., LIU, M., WEAVER, J. Framing Obesity: How News Frames Shape Attributions and Behavioral Responses. Journal of Health Communication: International Perspectives, Nova York, v. 21, n. 2, p. 139-147, 2016.

TEELE, D.L. Field Experiments and Their Critics: Essays on the Uses and Abuses of Experimentation in the Social Sciences. New Haven \& London: Yale University Press; 2014.

TURGEON, M.; CHAVES, B.S.; WIVES, W.W. Políticas de ação afirmativa e o experimento de listas: o caso das cotas raciais na universidade brasileira. Opinião Pública, Campinas, v. 20, n. 3, p. 363-376, 2014.

VASILJEVIC, M., PETRESCU, D. C., MARTEAU, T. M. Impact of advertisements promoting candy-like flavoured e-cigarettes on appeal of tobacco smoking among children: an experimental study. Tobacco Control. London, v. 25, n. E2, p. e107-e112, 2016.

ZHAO X, ALEXANDER TN, HOFFMAN L, JONES C, DELAHANTY J, WALKER M, BERGER AT, TALBERT E. Youth Receptivity to FDA's The Real Cost Tobacco Prevention Campaign: Evidence From Message Pretesting. Journal of Health Communication: International Perspectives, Nova York, v. 21, n. 11, p. 1153-1160, 2016. 


\section{Wladimir Gramacho}

Doutor em ciência política pela Universidade de Salamanca. Professor adjunto da Faculdade de Comunicação da Universidade de Brasília e coordenador do Centro de Pesquisa em Comunicação Política e Saúde Pública (FAC/UnB).

E-mail:wggramacho@gmail.com

\section{Emilly Ramos Behnke}

Bacharel em Jornalismo pela Universidade de Brasília e ex-bolsista do Programa de Iniciação Científica (Pibic/UnB). Atualmente, é repórter de política na sucursal de Brasília do jornal O Estado de S. Paulo e da Agência Estado. Anteriormente foi repórter da editoria de Cidades no jornal Correio Braziliense.

E-mail: emillybehnke@gmail.com

\section{Vitória Cavalcanti de Souza}

Graduanda em Publicidade e Propaganda pela Universidade de Brasília (UnB) e ex-bolsista do Programa de Iniciação Científica (Pibic/UnB). Atualmente trabalha como assessora de Comunicação e Processos Digitais na Deutsche Gesellschaft Für Internationale Zusammenarbeit ( $\mathrm{GIZ}$ ). Anteriormente, atuou como assistente de comunicação na Organização Internacional para Migrações (OIM).

E-mail: vitoria.souzacavalcanti@gmail.com 